\title{
Graphene Interfaced with Biological Cells: Opportunities and Challenges
}

\author{
Phong Nguyen and Vikas Berry* \\ Department of Chemical Engineering, Kansas State University, Manhattan, Kansas 66506, United States
}

ABSTRACT: By interfacing the quantum mechanical properties of nanomaterials with the complex processes in biology, several bio/nano systems have evolved with applications in biosensors, cellular devices, drug delivery, and biophotoluminescence. One recent breakthrough has been the application of graphene, a two-dimensional (2-D) sheet of $\mathrm{sp}^{2}$ hybridized carbon atoms arranged in a honeycomb lattice, as a sensitive platform for interfacing with biological cells to detect intra- and extracellular phenomena, including cellular excretion and cell membrane's potential modulation. In this Perspective, we discuss the recent results on graphene/cell interfacial devices and the principles defining the modulation of charge-carrier properties in graphene and its derivatives via interaction with cellular membranes. Graphene's high sensitivity in these applications evolves from the $\pi$-carrier cloud confined within an atom-thick layer, quantum-capacitance-induced doping enhancement, closely spaced electronic bands, and a large surface area. We discuss the effect of the electronegativity of the cell wall and the dynamic changes in its chemical potential on doping specific carriers into graphene. Finally, we discuss the challenges and opportunities of graphene-interfaced biocellular systems.

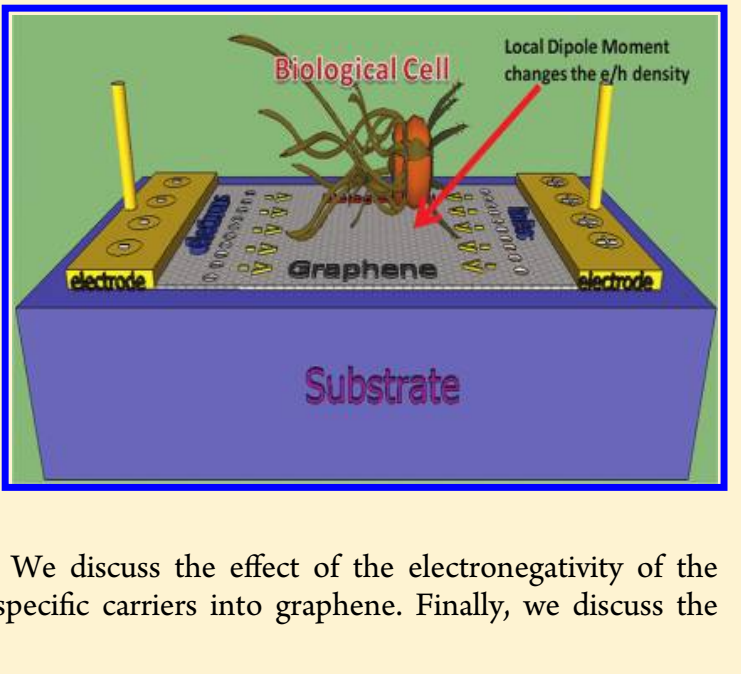

S ince the 1990s, several studies have originated on interfacing nanomaterials with biocomponents, with the goal of detecting biocomponents or biological phenomena. ${ }^{1-6}$ Until 2004, the research community was primarily applying zero-dimensional (0-D) and one-dimensional (1-D) nanomaterials (semiconducting nanoparticles, silicon nanowires), which are excellent for interfacing with biomolecules (DNA, proteins) and nanoscale biocomponents (viral particles, lipid micelles). These interfaces have led to the development of valuable tools and devices for biodiagnostics and biomedicine. $^{1,3,4,5}$ The 2004 experiments on graphene ${ }^{7}$ led to the evolution of 2-D nanotechnology. Owing to a unique combination of its crystallographic and electronic structure, ${ }^{8}$ graphene exhibits several superior and atypical properties, including weakly scattered $\left(\lambda_{\text {scattering }}>300 \mathrm{~nm}\right)$ ballistic transport of its charge carriers at room temperature, ${ }^{7,9,10}$ gate-tunable band gap in bilayers, $^{11}$ a chemically ${ }^{12,13}$ and geometrically ${ }^{14}$ controllable band gap, quantum Hall effect at room temperature, ${ }^{15,16}$ quantum interference, ${ }^{17}$ exceptional mechanical strength, ${ }^{18}$ and megahertz characteristic frequency. ${ }^{19}$ Due to these properties, graphene has emerged as an attractive candidate for several unique applications, including ultrafast nanoelectronic devices, ${ }^{8,10}$ single-molecule detectors, ${ }^{20}$ ultracapacitors, ${ }^{21}$ optoelectronics, ${ }^{22}$ and nanomechanical devices. ${ }^{19,23}$

\section{Graphene provides a sensitive platform for interfacing with biological cells to detect intra- and extracellular phenomena.}

In comparison to $0-\mathrm{D}$ nanoparticles ${ }^{1,2}$ and $1-\mathrm{D}$ nanowires, ${ }^{3-5}$ including their 2-D networks, ${ }^{6}$ the 2-D graphene sheets possess a large and continuous sensing/interfacing area. ${ }^{2}$ Therefore, graphene can provide a stable interface for microbes and mammalian cells, which too have large surface area (Figure 1).

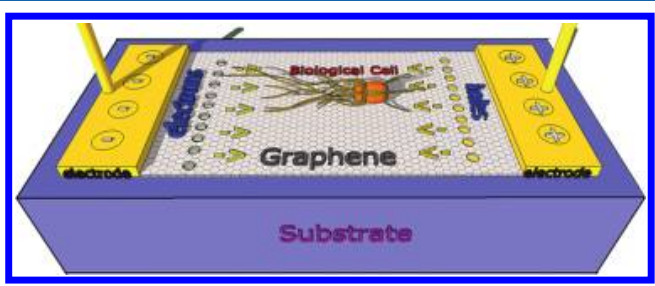

Figure 1. A generalized schematic for a graphene device interfaced with a biological cell.

Although several impedance-modulation mechanisms have been developed for cellular detection, these have primarily focused on the change in conduction through the cells. By interfacing with the cell, graphene provides an avenue to (a) change its own carrier properties via events on the cell wall, (b) interface strongly with the cell and interact with the cell wall by large surface area, (c) enable single-cell studies, and (d) maintain the cell's viability during measurements. ${ }^{24}$ The mechanism of the graphene/cell detection systems is based on carrier doping via the cell wall's electronegativity or dipole moment. Graphene's interface with the cell is via the cell wall or

Received: January 9, 2012

Accepted: March 29, 2012 
the cell membrane. Therefore, the cell's internal bioactivity's influence on the processes occurring on the cell membrane will stimulate the interfaced graphene device. For example, processes that can occur on the cell membrane include diffusion, osmosis, mediated transport, molecular engulfing, vesicle extrusion, ion channel conduction, and cell polarization (for example, in neuron cells). These processes will change the local dipole moment or apply a direct potential on the interfaced graphene.

\section{The mechanism of the graphene/ cell detection systems is based on carrier doping via the cell wall's electronegativity or dipole moment}

The attributes that enhance the sensitivity of graphene include the following: (a) Quantum capacitance: The quantumcoupling of the cell wall with graphene enhances the effective electric field due to the dipole moment of the interfacing cell wall $^{25}$ The quantum capacitance of graphene is given by $C_{\mathrm{q}}=$ $4 e \pi^{1 / 2} / h \vartheta_{\mathrm{F}}\left(n_{1}+n_{\mathrm{g}}\right)^{1 / 2}$, where $e$ is the electron charge, $h$ is Planck's constant, $\vartheta_{\mathrm{F}}$ is the Fermi velocity of the Dirac electron, and $n_{\mathrm{g}}$ and $n_{1}$ are the carrier concentrations from the gate potential and the intrinsic carrier concentration of graphene, respectively. The effective gating potential $\left(\Delta V_{\mathrm{G}}\right)$, therefore, translates from a change in dipole voltage $(\Delta V)$ of the cell wall to $\Delta V_{\mathrm{G}}=\left(C_{\mathrm{q}} / C_{\text {tot }}\right) \Delta V$, which is much higher than $\Delta V$. Here, $C_{\text {tot }}=\left(C_{\mathrm{q}}^{-1}+C_{\mathrm{g}}^{-1}\right)^{-1}$, and $\mathrm{C}_{\mathrm{g}}$ is the gate capacitance. (b) Confined doping: The change in the carrier concentration of graphene due to the change in the chemical potential of the cell wall is amplified as a result of the confinement of the doped carriers within graphene's ultrathin structure. (c) High charge mobility: Graphene sheets have a high carrier mobility, which amplifies the detection signal. (d) Surface area: Because graphene is a 2-D material, its surface conforms well along the cell wall. This enhances the sensitivity and the conductivity of the sensor. (e) Functionalization: Graphene can be functionalized with cell-wall-compatible biomolecules to further strengthen the interface with the cell. However, more work is required to ensure that graphene's characteristics are not altered as a result of its functionalization. The consortium of these properties makes it an ideal candidate for cellular detection.

In 2008, our group fabricated and studied bacteria-interfaced graphenic devices (see Figure 2). ${ }^{24}$ Here, the bacterial cells were detected on amine-functionalized graphene (GA), synthesized by diaminization of the immobilized graphene oxide (GO) sheets to produce chemically modified graphene (CMG). The device consisted of the GA deposited on a silica substrate and spanning two gold electrodes. The study was conducted for Gram positive Bacillus cereus cells possessing highly negatively charged surface due to the polyteichoic acid molecules on their cell wall. ${ }^{26,27}$ These negatively charged bacterial cells were electrostatically assembled on a positively charged GA sheet. ${ }^{26}$ Due to the multipoint electrostatic interfacing, the deposited bacteria illustrated a strong binding with GA and did not detach from the surface when washed with DI water at room temperature.

The binding of a single bacterium on the GA device exhibited a sharp $42 \%$ increase in conductivity. This is attributed to (a) the doping of a high density of holes on the p-type GA via its interfacing with the highly electronegative polyteichoic acid

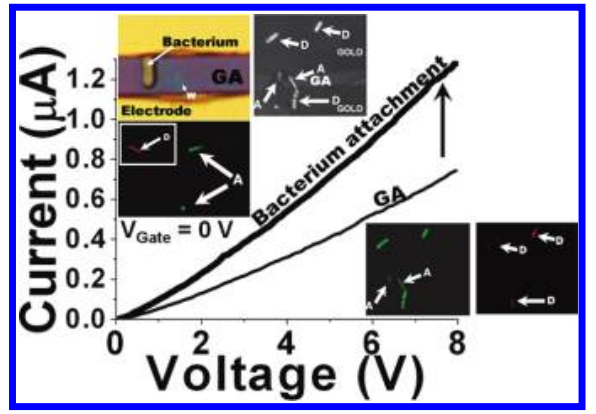

Figure 2. The conductivity of the p-type GA device increases upon attachment of a single bacterial cell on the surface of GA (top inset). Further, the cells are alive when attached. The bacteria on graphene were found to sustain the current and did not die, while the bacteria on the gold electrode died. ${ }^{24}$

groups on bacterium and (b) the additional holes being confined within the few-atom-thick conductive nanostructure (high surface to volume ratio), thus exhibiting a sensitive response. The hole density increase due to the bacterium attachment on GA was $3.53 \times 10^{10} \mathrm{~cm}^{-2}\left(\left.R_{1}\right|_{\mathrm{GA}}=10.85 \pm 0.51\right.$ $\left.\mathrm{M} \Omega,\left.R_{2}\right|_{\text {Bacteria }}=6.3 \pm 0.4 \mathrm{M} \Omega\right)$. This corresponds to a generation of $\sim 1400$ holes per bacterium in the GA. The electrical measurements did not have any visible effect on the integrity of the bacterium's structure as against the CNT network devices. ${ }^{6}$ The results showed a proof-of-concept of a highly sensitive graphene-based biodiagnostic tool with singlebacterium resolution.

In 2010, Zhang and $\mathrm{Chen}^{28}$ demonstrated that reduced graphene oxide (RGO) devices can be applied for detection of cellular secretion. In this device, live neuroendocrine PC12 cells were cultured directly on top of a poly-L-lysine-coated RGO transistor. This was followed by triggering the vesicular secretion of catecholamine molecules (dopamine, epinephrine, and norepinephrine). Here, the high $\mathrm{K}^{+}$solution was introduced into the recording chamber, which depolarized the cell membrane and allowed $\mathrm{Ca}^{2+}$ influx through the $\mathrm{Ca}^{2+}$ channels on the cell membrane. The change in the potential of the cellular membrane interfaced with the underlying RGO subsequently induced a device response shown by current spikes in the device (Figure 3). This was attributed to the vesicular release of catecholamines from a single PC12 cells. Here, the base RGO, a p-type semiconductor, is hole-doped via the electronegative catecholamine, thus undergoing an increase in conductivity. Further, the spike could be a result of catecholamine escaping into the solution. This study showed that a graphene-based field effect transistor (FET) can be highly sensitive to the dynamic cellular bioactivities atop graphenic surface.

While this field was developing, Lieber and Fang et al. ${ }^{29}$ showed that graphene devices can also be used to detect the change in the electrical potential (or "beating") of the cardiomyocyte cells (Figure 4). Here, the graphene FET device was interfaced with embryonic chicken cardiomyocytes (Figure 4, left B) cultured under optimized conditions on thin poly(dimethylsiloxane) (PDMS) sheets and transferred over the device via an $x-y-z$ manipulator. This brought a spontaneously beating cell into direct contact with the graphene sheet (Figure 4, right B). The cardiomyocyte cell atop an active graphene device exhibited regulated change in cell potential, which led to an ordered modulation of graphene's electrical properties, with the highconductivity peaks spaced with a frequency of $1.1 \mathrm{~Hz}$ and a signal-to-noise of $>4$. The high signal-to-noise ratio was 


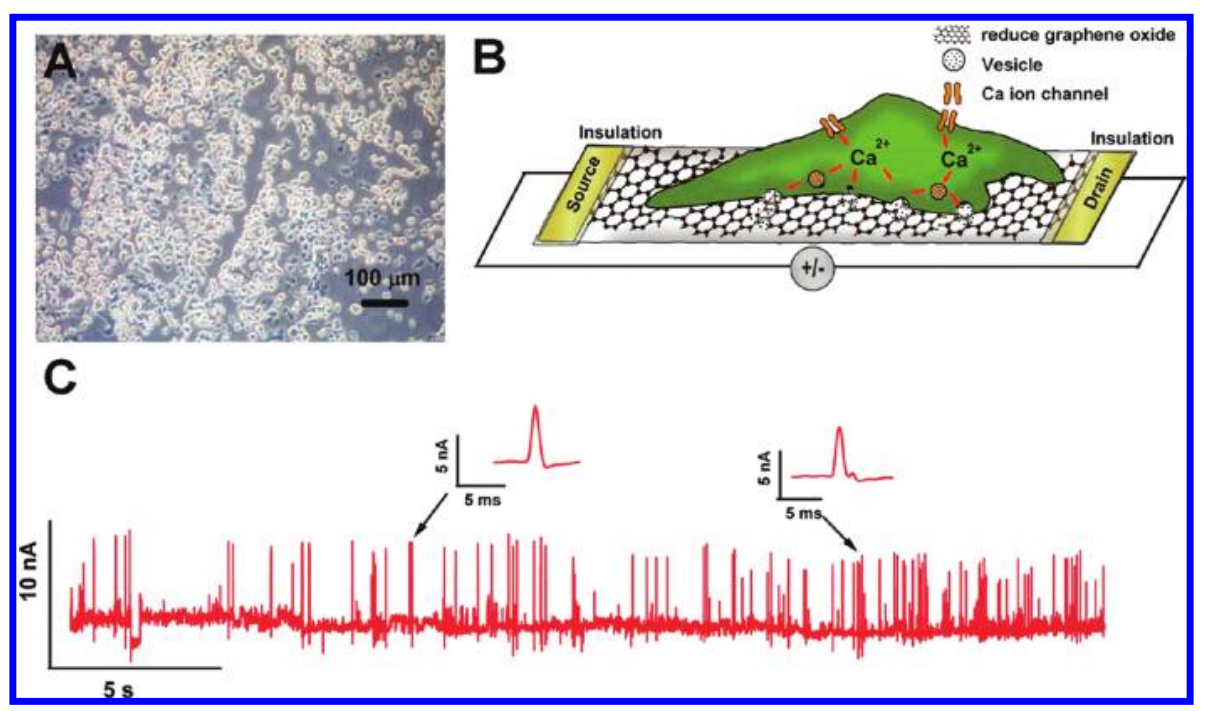

Figure 3. (A) Optical image of PC12 cells grown confluently on a poly-L-lysine-coated RGO device. (B) Schematic illustration of the interface between a PC12 cell and a RGO FET. (C) Real-time response of RGO to the vesicular secretion of catecholamines from PC12 cells stimulated by high $\mathrm{K}^{+}$solution. $V_{\mathrm{ds}}=100 \mathrm{mV}, V_{\mathrm{g}}=0 \mathrm{~V}$. The drain and source electrodes are $1 \mathrm{~cm}$ apart. ${ }^{28}$

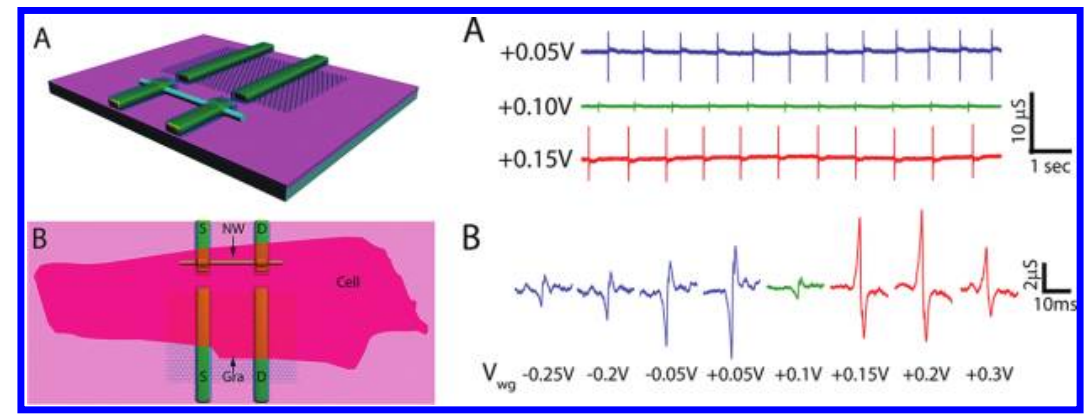

Figure 4. (Left) (A) Schematic of the graphene chip design. (B) Representation of the cardiomyocyte cell interfaced to typical graphene devices. (Right) (A) Temporal response of the graphene device at different water gate potentials (blue, green, and red traces are at $+0.05,+0.10$, and $+0.15 \mathrm{~V}$, respectively). The corresponding sensitivities are 2020, 398, and $2290 \mu \mathrm{S} / \mathrm{V}$, respectively. (B) Representative expanded peaks. Blue represents p-type graphene polarity, red peaks represent n-type graphene polarity, and the green peak was recorded near the Dirac point. ${ }^{29}$

attributed to the large interfacial area between graphene and the cell. Further, the magnitude of the conductance peak was also controlled by varying the gate voltage $\left(V_{\mathrm{wg}}\right)$. This device operation clearly showed that graphene is excellent for interfacing with mammalian cells and is sensitive to the electrogenics or the biochemical activity of the interfaced cell. This reinforces the impact of quantum-capacitance-induced enhancement of effective gating from the cell wall for these applications.

In addition, Hess and Garrido extended the above work by using large-area graphene grown by chemical vapor deposition (CVD). ${ }^{30}$ Here, the graphene device (Figure 5, top) could detect the electronic activity of the electrogenic cells ${ }^{29}$ more sensitively due to the higher charge carrier mobility and chemical stability of CVD graphene. In the fabricated device, cardiomyocyte-like HL-1 cells were cultured live directly on an array of graphenebased solution-gated transistors (G-SGFET). Here, the gate voltage was applied via electrolyte, thus controlling the Fermi level of graphene and changing the conductance across the device. The propagation of the surface potentials (action potential) across the cells exhibited the gate voltage spike signal of $900 \mu \mathrm{V}$ and a signal-to-noise ratio of 70 . Furthermore, the frequency of the voltage spikes was modulated by controlling the amount of fight-or-flight hormone, norepinephrine, in the media used to grow the cells. This study clearly shows the superiority of graphene for cellular-interfaced sensing devices with applications in neuroprosthetic devices and recording electrogenics activity.

In these graphene/cell devices, the generalized mechanism of modulation of graphene's carrier properties due to cell interfacing follows five steps. (I) The cell wall of the cellular entity interfaces with graphene, leading to a change in the local dipoles around graphene, which generates a change in the surface potential. A further change in the local dipoles can be caused by a change in the cell wall chemistry or potential due to cellular biophysical phenomena. (II) The effective gating potential due to this change is amplified via the quantum capacitance of graphene $\left(\Delta V_{\mathrm{G}}=\left(C_{\mathrm{q}} / C_{\text {tot }}\right) \Delta V\right)$, as mentioned earlier. (III) The density of carriers in graphene is modulated as a response (doping). The semimetallic nature of graphene enables direct interface-dipole-induced doping of the carriers. ${ }^{15,31}$ (IV) The confinement of the $\pi$-carriers within a few atoms ${ }^{7}$ ensures that the change in the interfacial potential directly and efficiently modulates graphene's carrier density. This is because the modulation of the number of charge carriers is not distributed over a large thickness (or volume). (V) The transport of the carriers occurs. The $\pi$-orbitals in graphene are atop its lattice plane; thus, the carriers experience low scattering, 


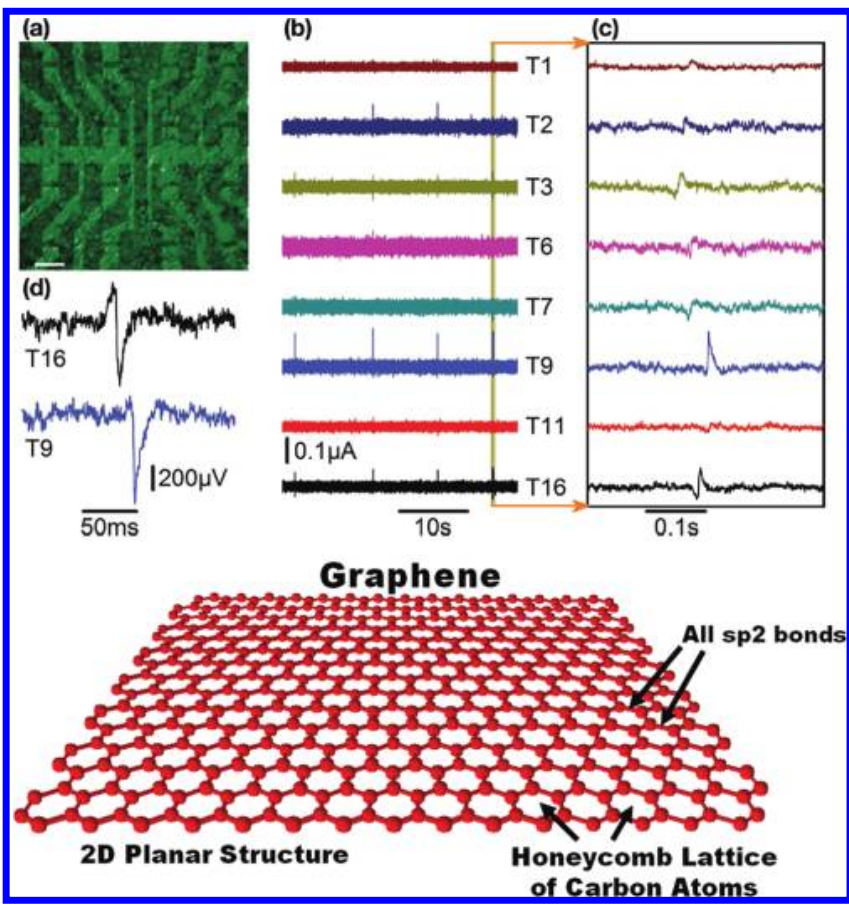

Figure 5. (Top) (a) Optical micrograph of a transistor array. Scale bar $=$ $100 \mathrm{~m}$. (b,c) Current recordings of eight transistors in one FET array. (d) Exemplary single spikes with current response converted to extracellular voltage signal. The upper spike resembles a capacitive coupling followed by the opening of voltage-gated sodium channels, whereas in the bottom one, the ion channels dominate over the capacitive coupling. Reproduced with permission of Wiley- $\mathrm{VCH}$, copyright $2011 .^{29}$ (Bottom) Graphene lattice structure: $\mathrm{sp}^{2}$ hybridized carbon atoms arranged in a $2 \mathrm{D}$ honeycomb lattice.

exhibiting high mobility. Therefore, a highly amplified sensor signal $\left(\Delta I / V_{\mathrm{DS}} \propto n A \mu\right)$ is realized, which enables sensitive detection of the interfacial event. (VI) At the molecular scale, the interfacial event on graphene's surface can also introduce scattering sites and/or electrically gate the carriers on graphene, ${ }^{24}$ which can also modulate its electrical properties. The change in carrier density $(\Delta q)$ can be measured by the difference in the resistivity before and after the interfacial event.

$$
\begin{aligned}
& \mu_{\text {Carrier }}=\left(\Delta I_{\mathrm{DS}} / \Delta V_{\mathrm{G}}\right) /\left(C(l / w) V_{\mathrm{DS}}\right) \\
& \Delta q=\left(R_{2}^{-1}-R_{1}^{-1}\right) /\left((l / w) \mu_{\text {Carrier }}\right) \quad \Delta q=C \Delta V_{\text {Dirac }}
\end{aligned}
$$

We opinionate that the current major challenge in the advancement of graphene biocellular devices is graphene's functionalization with specific bioanchors without altering its electrical properties. Functionalization is important for two reasons, (a) it adds specificity to the event being detected, which enhances the magnitude of the desired signal, and (b) it improves the interface between the cell and graphene. Our group has recently shown that graphene functionalized with concanavalin, a pectin protein with high specific affinity to the bacterial cell wall (Gram positive), can cause it to hermetically wrap bacterial cells. ${ }^{32}$ The functionalization dramatically improved graphene's interaction with the cell wall. Here, the graphenic wrapping acts as an impermeable electron transparent encasement, allowing real wet imaging of the cells. However, this functionalization process converts the $\mathrm{sp}^{2}$ hybridized state of the carbon atoms to $\mathrm{sp}^{3}$ and thus reduces its carrier mobility and sensitivity. This implies that a nondestructive functionalization, which does not convert the $\mathrm{sp}^{2}$ hybridization state of the carbon atoms in graphene, can have significant impact on the cell-interfaced graphene devices. Traditional routes like Hummers method based oxidation, plasma-induced functionalization, and nanoparticle incorporation lead to significant $\mathrm{sp}^{3}$ hybridization, reduction in $\pi$-carrier density, and introduction of scattering sites. An alternative route for nondestructive functionalization of graphene is $\pi-\pi$ interfacing. Here, $\pi-\pi$ bonded molecules on graphene do not disturb the $\mathrm{sp}^{2}$ hybridization state of the carbon atoms on graphene, such that the high mobility of graphene is retained. Therefore, more efforts are required toward functionalizing graphene via $\pi-\pi$ bonds to enhance the specificity and interfacial strength in graphene's biocellular device applications.

\section{While the chemical modification is expected for improved specificity, structural modification of graphene can further improve its sensitivity.}

While the chemical modification is expected to improve specificity, structural modification of graphene can further enhance its sensitivity. For example, the opening up of a band gap with electrical gating in bilayer graphene ${ }^{11}$ makes it a more sensitive system than single-layer graphene for bioelectronic sensors. It is expected that cellular interfaces with bilayer graphene will be able to detect lower-order signals more effectively via bioelectrical gating. This is expected to improve the signal-to-noise ratio in the detection of electrogenic activity of cardiomyocytes. Further, thin films of graphene nanoribbons (GNRs) will also provide an important opportunity for biointerfacing, where the band gap of GNRs can enhance the detection sensitivity. Here, the band gap due to quantum confinement will produce a barrier for the carriers, which will further increase the sensitivity of the device. Similarly, graphene with punched holes (or graphene nanomesh ${ }^{33}$ ) can also be used as a sensitive graphenic substrate with a band gap for biocellular interfacing and detection. Graphene nanomesh can be fabricated via transferring the film morphology of block copolymers on graphene via lithography.

Because graphene can strongly interface with cells, another opportunity that graphene/cell interfaces provides is production of energy from electrochemically active cells. For example, geobacter is a family of bacteria, which produces protons on its cell wall as a byproduct of metabolism. ${ }^{34}$ These geobacter cells have been used to produce power in several biobattery devices. ${ }^{34}$ It is expected that the large area interfacing with these cells will enhance the efficiency of the battery. Graphene can provide a significantly enhanced interface to these cells to draw electricity effectively. However, this will require dopant engineering to achieve high charge separation with low recombination probability.

Further, the following sensor studies are required for further characterization of these graphene biocellular devices: (i) Specificity has to be evaluated by the $F$ measure $\left(F=\left[\left(1+\beta^{2}\right)\right.\right.$ $(\operatorname{Pr} \times \operatorname{Re})] /\left(\beta^{2} \operatorname{Pr}+\operatorname{Re}\right)$, where $\beta, \operatorname{Pr}$, and $\operatorname{Re}$ are the $F$ parameter, precision, and recall, respectively). (ii) Device resolution: The device resolution can be measured via simultaneous signal acquisition while incrementally increasing the intensity of the interfacial event. The event detection resolution measures the smallest unit defining the interfacial event, 
which can produce a discernible change in the signal. (iii) Temperature effects: The effect of temperature on the event detection characteristics also needs to be studied for these devices.

In summary, this Perspective article outlines the recent developments in graphene-based biocellular devices and discusses the electrical and structural characteristics of graphene, which are leveraged in biosensing and biointerfacial applications. We outline a generalized mechanism of graphenebased cellular devices and identify the lack of specificity as the major bottleneck in the development of this field. Further, the sensitivity of the devices can be improved by using graphene bilayers, graphene nanoribbon films, or graphene nanomeshbased platforms. We envision that cell-interfaced graphene devices can open avenues for biocellular batteries (geobacter), biodiagnostics and single-cell analysis.

\section{The sensitivity of graphene-based biocellular devices can be improved by using graphene bilayers, graphene nanoribbon films, or graphene nanomesh-based platforms.}

\section{AUTHOR INFORMATION}

\section{Corresponding Author}

*E-mail: vberry@ksu.edu.

\section{Notes}

The authors declare no competing financial interest.

\section{Biographies}

Phong Nguyen received his B.S. from Emporia State University and is currently a Ph.D. candidate in the Department of Chemical Engineering at Kansas State University. His current research interests are in the areas of graphene-based detectors for molecular motion, nanolithography, and large-area graphene synthesis and functionalization.

Vikas Berry is the William H. Honstead Professor of Chemical Engineering and assistant professor at Kansas State University. $\mathrm{He}$ received his Ph.D. from Virginia Tech in 2006, M.S. from KU in 2002, and B.S. from IIT-Delhi in 1999. Amongst his honors is the NSFCAREER award. His current areas of research interest include graphene science and technology, bionanotechnology, and molecular electronics. (http://www.che.ksu.edu/ vberry/index.html).

\section{ACKNOWLEDGMENTS}

V.B. thanks the financial support from NSF (CMMI-1054877, CMMI-0939523, and CMMI-1030963), the Office of Naval Research (Grant N000141110767) Terry C. Johnson Center for Basic Cancer Research, and a KSU start-up.

\section{REFERENCES}

(1) Cai, H.; Xu, C.; He, P. G.; Fang, Y. Z. Colloid Au-Enhanced DNA Immobilization for the Electrochemical Detection of SequenceSpecific DNA. J. Electroanal. Chem. 2001, 510, 78-85.

(2) Le, J. D.; Pinto, Y.; Seeman, N. C.; Musier-Forsyth, K.; Taton, T. A.; Kiehl, R. A. DNA-Templated Self-Assembly of Metallic Nanocomponent Arrays on a Surface. Nano Lett. 2004, 4, 2343-2347.
(3) Cai, H.; Cao, X. N.; Jiang, Y.; He, P. G.; Fang, Y. Z. Carbon Nanotube-Enhanced Electrochemical DNA Biosensor for DNA Hybridization Detection. Anal. Bioanal. Chem. 2003, 375, 287-293.

(4) Cui, Y.; Wei, Q. Q.; Park, H. K.; Lieber, C. M. Nanowire Nanosensors for Highly Sensitive and Selective Detection of Biological and Chemical Species. Science 2001, 293, 1289-1292.

(5) Patolsky, F.; Zheng, G. F.; Lieber, C. M. Nanowire-Based Biosensors. Anal. Chem. 2006, 78, 4260-4269.

(6) So, H. M.; Park, D. W.; Jeon, E. K.; Kim, Y. H.; Kim, B. S.; Lee, C. K.; Choi, S. Y.; Kim, S. C.; Chang, H.; Lee, J. O. Detection and Titer Estimation of Escherichia coli Using Aptamer-Functionalized Single-Walled Carbon-Nanotube Field-Effect Transistors. Small 2008, 4, 197-201.

(7) Novoselov, K. S.; Geim, A. K.; Morozov, S. V.; Jiang, D.; Zhang, Y.; Dubonos, S. V.; Grigorieva, I. V.; Firsov, A. A. Electric Field Effect in Atomically Thin Carbon Films. Science 2004, 306, 666-669.

(8) Novoselov, K. S.; Jiang, D.; Schedin, F.; Booth, T. J.; Khotkevich, V. V.; Morozov, S. V.; Geim, A. K. Two-Dimensional Atomic Crystals. Proc. Natl. Acad. Sci. U.S.A. 2005, 102, 10451-10453.

(9) Zhou, S. Y.; Gweon, G. H.; Graf, J.; Fedorov, A. V.; Spataru, C. D.; Diehl, R. D.; Kopelevich, Y.; Lee, D. H.; Louie, S. G.; Lanzara, A. First Direct Observation of Dirac Fermions in Graphite. Nat. Phys. 2006, 2, 595-599.

(10) Novoselov, K. S.; Geim, A. K.; Morozov, S. V.; Jiang, D.; Katsnelson, M. I.; Grigorieva, I. V.; Dubonos, S. V.; Firsov, A. A. TwoDimensional Gas of Massless Dirac Fermions in Graphene. Nature 2005, 438, 197-200.

(11) Zhang, Y.; Tang, T. T.; Girit, C.; Hao, Z.; Martin, M. C.; Zettl, A.; Crommie, M. F.; Shen, Y. R.; Wang, F. Direct Observation of a Widely Tunable Bandgap in Bilayer Graphene. Nature 2009, 459, 820-823.

(12) Gilje, S.; Han, S.; Wang, M.; Wang, K. L.; Kaner, R. B. A Chemical Route to Graphene for Device Applications. Nano Lett. 2007, 7, 3394-3398.

(13) Gomez-Navarro, C.; Weitz, R. T.; Bittner, A. M.; Scolari, M.; Mews, A.; Burghard, M.; Kern, K. Electronic Transport Properties of Individual Chemically Reduced Graphene Oxide Sheets. Nano Lett. 2007, 7, 3499-3503.

(14) Barone, V.; Hod, O.; Scuseria, G. E. Electronic Structure and Stability of Semiconducting Graphene Nanoribbons. Nano Lett. 2006, $6,2748-2754$.

(15) Novoselov, K. S.; Jiang, Z.; Zhang, Y.; Morozov, S. V.; Stormer, H. L.; Zeitler, U.; Maan, J. C.; Boebinger, G. S.; Kim, P.; Geim, A. K. Room-Temperature Quantum Hall Effect in Graphene. Science 2007, $315,1379$.

(16) Novoselov, K. S.; McCann, E.; Morozov, S. V.; Fal'ko, V. I.; Katsnelson, M. I.; Zeitler, U.; Jiang, D.; Schedin, F.; Geim, A. K. Unconventional Quantum Hall Effect and Berry's Phase of $2 \pi$ in Bilayer Graphene. Nat. Phys. 2006, 2, 177-180.

(17) Young, A. F.; Kim, P. Quantum Interference and Klein Tunnelling in Graphene Heterojunctions. Nat. Phys. 2009, 5, 222226.

(18) Lee, C.; Wei, X. D.; Kysar, J. W.; Hone, J. Measurement of the Elastic Properties and Intrinsic Strength of Monolayer Graphene. Science 2008, 321, 385-388.

(19) Bunch, J. S.; van der Zande, A. M.; Verbridge, S. S.; Frank, I. W.; Tanenbaum, D. M.; Parpia, J. M.; Craighead, H. G.; McEuen, P. L. Electromechanical Resonators from Graphene Sheets. Science 2007 315, 490-493.

(20) Schedin, F.; Geim, A. K.; Morozov, S. V.; Hill, E. W.; Blake, P.; Katsnelson, M. I.; Novoselov, K. S. Detection of Individual Gas Molecules Adsorbed on Graphene. Nat. Mater. 2007, 6, 652-655.

(21) Stoller, M. D.; Park, S.; Zhu, Y.; An, J.; Ruoff, R. S. GrapheneBased Ultracapacitors. Nano Lett 2008, 8, 3498-3502.

(22) Liu, Q.; Liu, Z. F.; Zhang, X. Y.; Zhang, N.; Yang, L. Y.; Yin, S. G.; Chen, Y. S. Organic Photovoltaic Cells Based on an Acceptor of Soluble Graphene. Appl. Phys. Lett. 2008, 92, 223303.

(23) Poot, M.; van der Zant, H. S. J. Nanomechanical Properties of Few-Layer Graphene Membranes. Appl. Phys. Lett. 2008, 92, 06311. 
(24) Mohanty, N.; Berry, V. Graphene-Based Single-Bacterium Resolution Biodevice and DNA Transistor: Interfacing Graphene Derivatives with Nanoscale and Microscale Biocomponents. Nano Lett. 2008, 8, 4469-4476.

(25) Xia, J.; Chen, F.; Li, J.; Tao, N. Measurement of the Quantum Capacitance of Graphene. Nat Nano 2009, 4, 505-509.

(26) Berry, V.; Saraf, R. F. Self-Assembly of Nanoparticles on Live Bacterium: An Avenue to Fabricate Electronic Devices. Angew. Chem., Int. Ed. 2005, 44, 6668-6673.

(27) Berry, V.; Gole, A.; Kundu, S.; Murphy, C. J.; Saraf, R. F. Deposition of CTAB-Terminated Nanorods on Bacteria to Form Highly Conducting Hybrid Systems. J. Am. Chem. Soc. 2005, 127, 17600-17601.

(28) He, Q.; Sudibya, H. G.; Yin, Z.; Wu, S.; Li, H.; Boey, F.; Huang, W.; Chen, P.; Zhang, H. Centimeter-Long and Large-Scale Micropatterns of Reduced Graphene Oxide Films: Fabrication and Sensing Applications. ACS Nano 2010, 4, 3201-3208.

(29) Cohen-Karni, T.; Qing, Q.; Li, Q.; Fang, Y.; Lieber, C. M. Graphene and Nanowire Transistors for Cellular Interfaces and Electrical Recording. Nano Lett. 2010, 10, 1098-1102.

(30) Hess, L. H.; Jansen, M.; Maybeck, V.; Hauf, M. V.; Seifert, M.; Stutzmann, M.; Sharp, I. D.; Offenh+ñusser, A.; Garrido, J. A. Graphene Transistor Arrays for Recording Action Potentials from Electrogenic Cells. Adv. Mater. 2011, 23, 5045-5049.

(31) Geim, A. K.; Novoselov, K. S. The Rise of Graphene. Nat. Mater. 2007, 6, 183-191.

(32) Mohanty, N.; Fahrenholtz, M.; Nagaraja, A.; Boyle, D.; Berry, V. Impermeable Graphenic Encasement of Bacteria. Nano Lett. 2011, 11, $1270-1275$.

(33) Bai, J.; Zhong, X.; Jiang, S.; Huang, Y.; Duan, X. Graphene Nanomesh. Nat. Nano 2010, 5, 190-194.

(34) Bond, D. R.; Lovley, D. R. Electricity Production by Geobacter sulfurreducens Attached to Electrodes. Appl. Environ. Microbiol. 2003, $69,1548-1555$. 